\title{
Direct Optimization of an Automotive Sheet Metal Part Using ANSYS
}

\author{
U. Khalid ${ }^{1}$, O. Mustafa 2 , M. A. NAEEM ${ }^{3}$, M. AlKhateeb ${ }^{4}$, B.AWAD ${ }^{5}$ \\ ${ }^{1}$ University of Debrecen, Faculty of Engineering, Department of Mechanical Engineering, \\ ukajk@live.com \\ ${ }^{2}$ University of Debrecen, Faculty of Engineering, Department of Mechanical Engineering, \\ Othman.mustafa93@gmail.com \\ ${ }^{3}$ University of Debrecen, Faculty of Engineering, Department of Mechanical Engineering, \\ m.alinaeem94@gmail.com \\ ${ }^{4}$ University of Debrecen, Faculty of Engineering, Department of Mechanical Engineering, \\ mohammad5atib@gmail.com \\ ${ }^{5}$ University of Debrecen, Faculty of Engineering, Department of Mechanical Engineering, \\ basel_marwan@icloud.com
}

\begin{abstract}
Optimization of automotive parts nowadays is mainly used to design lightweight and cost-effective vehicle parts in order to improve the cost and efficiency. In this research, a sheet metal part was taken into consideration and optimized using direct optimization module in ANSYS to evaluate the process. An initial Finite Element Analysis (FEA) was done on the sheet metal part by adding forces and constraints in order to initiate direct optimization. The purpose of the optimization is to minimize the mass of the sheet metal part and maintaining a certain Factor of Safety (FOS) by automatically modifying the sheet thickness and the dimension of the side holes. As a result, the best candidate point with 23\% mass reduction was found which complied with FOS value was selected for optimal geometry.
\end{abstract}

Keywords: Optimization, ANSYS, Sheet-metal, Structural Optimization, Finite Element Analysis

\section{Introduction}

In any process of product development, designing phase is of paramount importance since it dictates the costs, time and methods associated with the product [1]. Nowadays, increased competition is forcing companies to look for new development ways and engineers are required to look for the right balance between product performance, cost and production time [2]. Therefore, there is a need to make this phase more efficient through design optimization. Optimization can be defined as a rational search for the optimal spatial distribution of material within a prescribed admissible structural domain [3]. The structural optimization has three types; shape optimization, size optimization and topology optimization [4]. They all change different parameters of the geometry, shape optimization finds the optimal shape that minimizes a certain cost function while satisfying the constraints while size optimization varies just the size to do so. The topology optimization is defined as a process which is iterative and consists of the metamorphosis of the design space into an innovative, complex and highperformance structure [5]. Mathematically, it is the minimization or maximization of a function subject 
to constraints on its variables [6]. The process of optimization is of vital importance in the industry, helping them to meet the market competitiveness and fuel economy regulations.

CAD forms the backbone of modern industries since it represents complex mechanical problems in a simpler way, making it easier for engineers to account for all the factors affecting the study. Through different versions of CAD software, it is possible to simulate all the phases of product development before it is put to real test resulting in reduced costs and failure. Traditional CAD methods rely on the imagination and knowledge of the designer, where manual optimization is carried out by making multiple concept solutions and evaluated to determine which best fits the design goals [7]. This is potentially suboptimal and does not lead to complete understanding of the problem. Nowadays, new optimization functions built within the CAD software use certain evolutionary algorithms which use an analogy with natural evolution to perform search by evolving solutions to problems [8]. These algorithms search for optimal solution rather than using simulation approach to study limited number of configurations. Optimization gives the right material distribution and sizing within the design space to achieve the design objectives. Three types of structural optimizations are shape, size and topology optimization. Gunwant, D., \& Misra, A. (2012) used topology optimization in ANSYS on four rectangular sheet metals to find out the optimal layout of material in brackets. The result was a truss like structure which can be optimised further for weight reduction [9]. This transformation from sheet metal to truss shows the importance of material distribution methods and how drastic of an effect it can have on final design. Another main approach in structural optimization techniques is homogenization method in which a material model with micro-scale voids is processed to seek optimal porosity. It is capable of producing internal holes in the geometry without prior knowledge of their existence [10].

ANSYS topology optimization makes it easier to design durable light weight components for different applications, defining the objectives easily and applying control parameters which fulfil the manufacturing requirements. Direct optimization in ANSYS offers several methods for single and multiobjective optimization based on the input and output parameters, the correlations between input and output parameters can be identified in order to study and control the desired output which might be cost, quality or process time. Small changes in the input lead to significant changes in the output; hence the optimization process is highly sensitive to the initial parameters as shown in study [9].

The optimization process uses certain algorithms to find the ideal solution and these algorithms are inspired by biological evolution. They are called evolutionary algorithms and the ones used for optimization include particle swarm optimization (PSO), simulated annealing (SA), Genetic Algorithms (GAs) and simulated biological growth (SBG) [11]. The best accepted method of them all is GA (Goldberg 1989; De Jong 1975) [12] which relies on the concept of survival of the fittest. It is similar to the process of natural evolution to search by evolving solutions to the problem based on the collective learning process within a population of individuals, each representing a search point in the space of possible solutions [13]. There is a large sample of solutions and feasibility of each solution is evaluated as they improve through natural selection caused by environmental changes. The solutions yielding better results are more refined through cross-over reproduction, giving more appropriate solutions. The process of evaluation, natural selection and reproduction continues until it converges to a satisfactory solution. 
The MOGA method (Multi-Objective Genetic Algorithm) is a multi-objective optimization method available in ANSYS. It is a method that achieves multi-objective optimization using genetic algorithms. Multi-objective optimization usually involves having multiple objective functions and optimizing multiple parameters at the same time. It has been applied to many fields including: Economics, finance and Engineering. Usually Engineers are expected to make choices related to conflicting objectives. An example of such decisions is trying to maximize a car performance and speed while also trying to reduce fuel consumption and emissions of pollutants. Multiple solutions exist for such problems and there are many types of methods in order to handle the set of solutions. These methods may include converting the problem into a single solution problem or including an experienced decision maker to select the best possible solution. The MOGA method uses genetic algorithms to find the best possible set of solutions of the multi-object optimization problem and offers multiple candidate solutions for the user to decide the best one. MOGA has been used to solve multiple problems over the years. This method was used in a study for the optimization of a super plastic forming process where the input parameters that were chosen were the sample diameter and thickness. This study highlighted the influence of inputs on the outputs, and prediction of the shape of product, optimal initial geometry, cracks and fracture [14]. There has been research on the use of MOGA in the design of pressure swing absorption, the effect of the parameters of the MOGA was studied and a comparison was made with different search methods to determine the efficiency of the algorithm [15]. Evaluation of the effectiveness of MOGA and DSMs (direct search methods) showed that MOGA is more reliable, efficient and do a good approximation of the problem under focus as compared to DSMs.

There have been studies on optimization process of rubber product designs where a different non-linear FEA has been used since rubber can suffer from large deformations upon stress [16] [17]. Different FEM models have been introduced for an accurate shape optimization of rubber bodies using a rubber bumper as an experimental object where the load displacement curve was mapped considering all affecting factors [18] [19]. The mathematical model of these rubber bodies have shown to be very different from normal due to their elasticity. One study [20] also utilizes a special purpose FEM code written in FORTAN for the analysis of nearly incompressible rubber parts. It aimed to reduce computation time and optimize the shape of rubber part under investigation. This along with another study [21] presented regression functions which can accurately describe the relation between input and output data of a complex technical problem such as of rubber parts under changing loads.

In a study conducted by Hafiz, A. A. M., (2019), they modelled a sheet metal forming process using finite element method to find out the critical parameters and to avoid failure or defective products. Procedure of die design was topologically optimized to enhance the die tool shapes based on the finite element analysis. The reduction of mass and change in the final shape lead to reduced defects, less stress concentration and an accurate simulation of the blanking procedure [22]. Similarly, Prasanna P. et al., (2017) carried out a stress study of a pressure vessel using ANSYS software and critical pressure points of the wall were analysed. Then an optimized geometry is modelled to overcome the stresses produced in the vessel. The results have showed that the stress values in the modified model were better than the actual model since the value of the stress has decreased which can lead to an increase in the life time of the vessel material [23]. Similar areas of performance will be explored in this study to present the degree of effect optimization has on the geometry of a sheet metal part and its performance overall. We will evaluate in depth the "Direct Optimization" tool in DesignXplorer using ANSYS software. 


\section{Materials and Method}

The sheet metal part geometry chosen for this study is shown [Fig. 1] along with the material chosen which is an Aluminium Alloy with properties as in [Fig. 2]. The amount of force applied on the hinges combined is $1414 \mathrm{~N}$ and the direction of force, constraints and variables are also labelled in this figure. Finite element analysis of the initial body that gives the total deformation and stress contours was carried out with two qualities of meshes. One type of mesh was the default low quality one which was then customized for element size value (approaching 1) and skewness to improve FEA results. The maximum stress was found to be $100.5 \mathrm{MPa}$ for the improved mesh which is a more accurate value [Fig. 2].

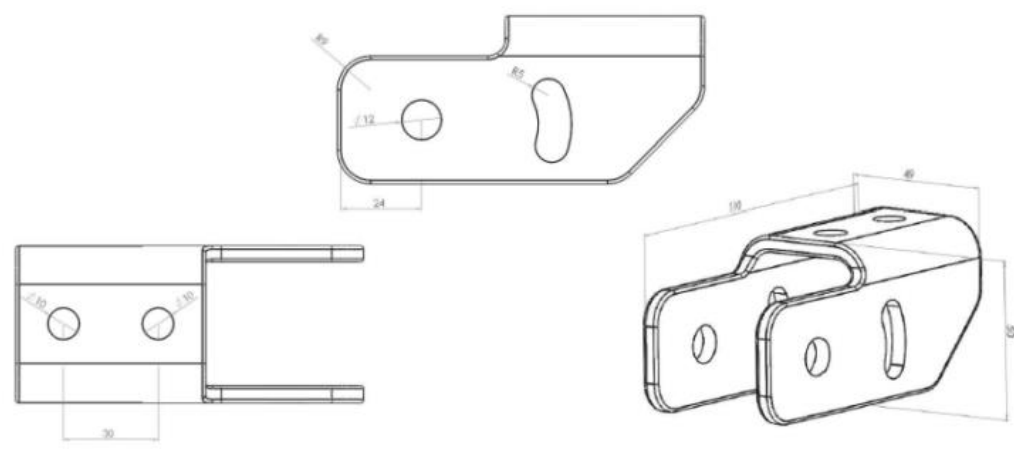

Figure 1: Initial Dimensions

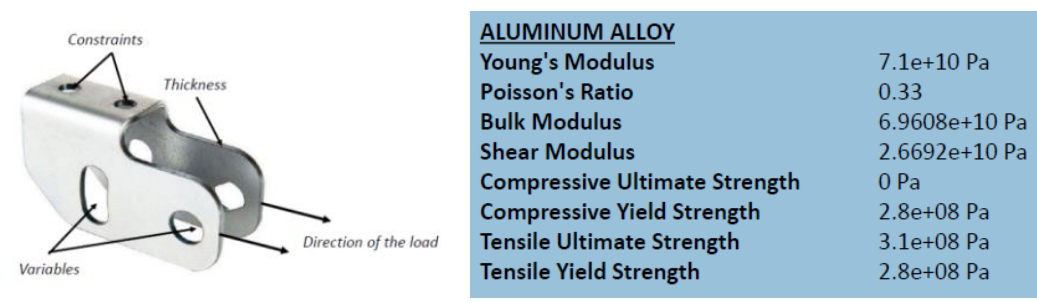

Figure 2: Initial geometry with labelling and its material properties
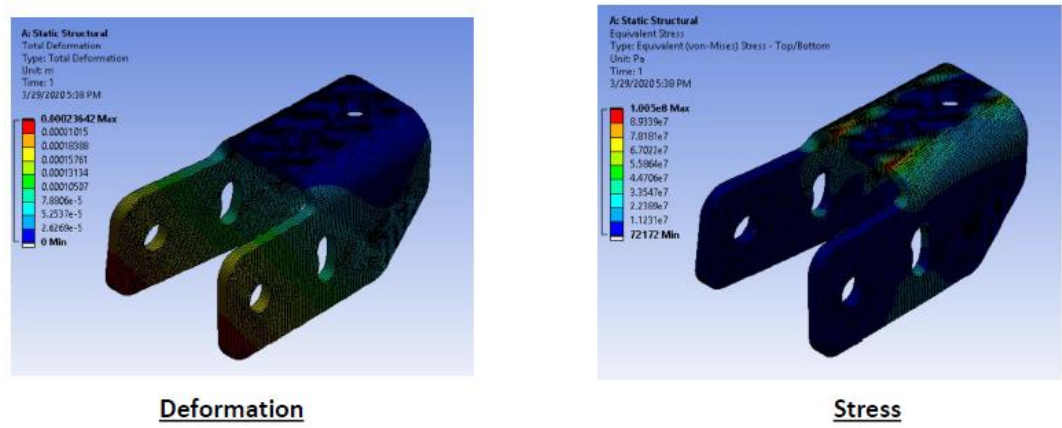

Figure 3: Initial body FEA results

We are targeting the design to have a factor of safety of 1.5 or which means maximum allowable stress can be $186 \mathrm{MPa}$, comparing that with the current value of 100MPa shows us that there is plenty of room for optimization through mass reduction. The optimization module used in this research is the direct optimization method in ANSYS. The initial optimization was done with 11 sample points which was then rerun using 50 sample points. Three candidate points which best adhered to the FOS 1.5 value were 
chosen and simulated [fig. 4]. The input parameters, thickness of sheet and diameter of side holes were kept in the range of $(3.0-5.5 \mathrm{~mm})$ and $(11-14 \mathrm{~mm})$.

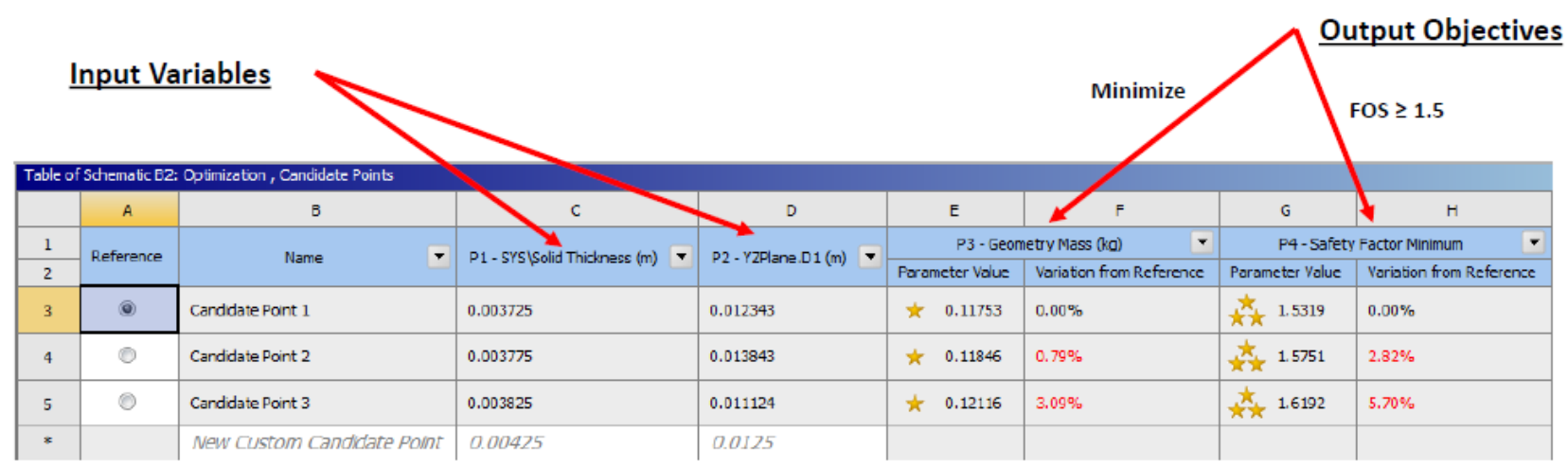

Figure 4: Three candidate points obtained from optimization using 50 sample points

\section{Results}

The optimization has been carried out and the obtained data and candidate points have been analysed to find out the optimum input data points in order to decrease the mass and material costs of this part. The candidate points resulting from all the 50 iterations including the three best candidates have been shown in [fig. 5]. The three black coloured lines indicate the three best candidate points which have been further analysed to reach an end result. Pink lines represent acceptable candidate points as they join the right side of the graph at FOS value of above 1.5 while grey lines represent the rejected ones being in non-compliance.

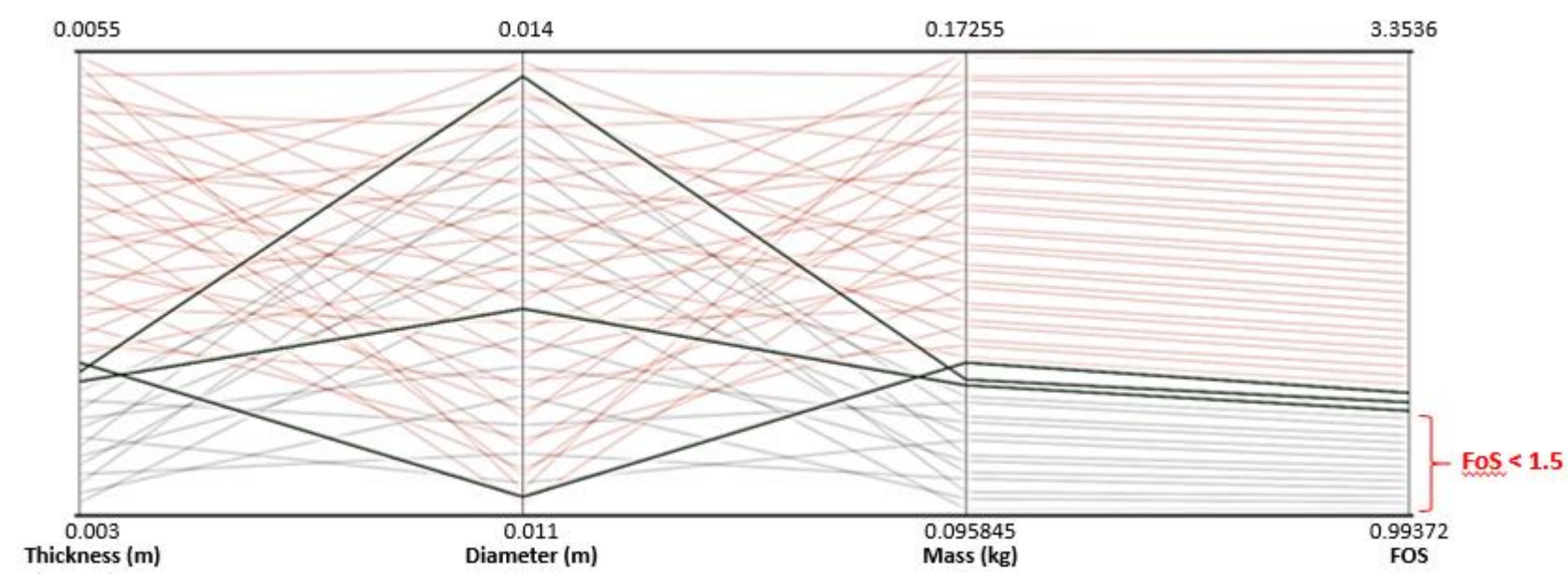

Figure 5: Three candidate points

The optimized geometry with new values of dimensional variables is shown in [fig. 6]. It has dimensions in values up to 3 decimal points which require high precision machining increasing the manufacturing costs. These values are rounded off to the nearest whole number for the ease of manufacturing as seen in [fig. 7]. This rounding off increases the mass by a small margin again but it's worth doing so considering the costs of high precision CNC machining. This concept of optimization can be extended 
beyond this study to apply on designing of any sheet metal parts such as the ones in automotive industry where they exist in larger sizes. Reducing mass of such parts would add up and in the end lead to significant cost cuts of production.
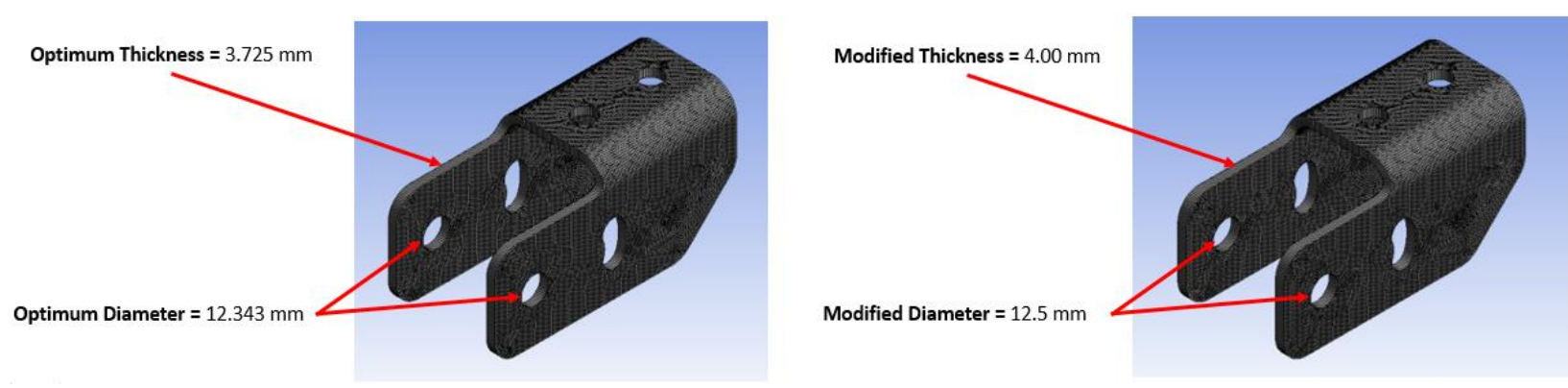

Figure 6: Optimized geometry

Figure 7: Optimized geometry with rounded off dimension values

The direct optimization gave us 3 candidate points to choose from in order to study and analyse the effect of the input parameters on the output objectives. Candidate point 1 which corresponds to $3.725 \mathrm{~mm}$ sheet thickness and $12.34 \mathrm{~mm}$ of hole diameter can be considered as the optimal solution since it complies with FOS 1.5 criteria while keeping minimum size resulting in minimum mass. The new value of sheet metal thickness shows a decrease of nearly $25 \%$ from the original value while the diameter value increased by almost $3 \%$, contributing to reduction of mass overall. The mass of optimized design has decreased by nearly $23 \%$ to a value of $0.12343 \mathrm{~kg}$. Finite element analysis of the newly optimized model was carried out to confirm its adherence to the design limits [fig. $8 \& 9$ ]. We can see the maximum stress value $182 \mathrm{MPa}$ is very close to the limit $186 \mathrm{MPa}$ in original optimized body since its purely optimal but after the dimension values are rounded off, the mass increases and maximum stress becomes less i.e. $157 \mathrm{MPa}$.
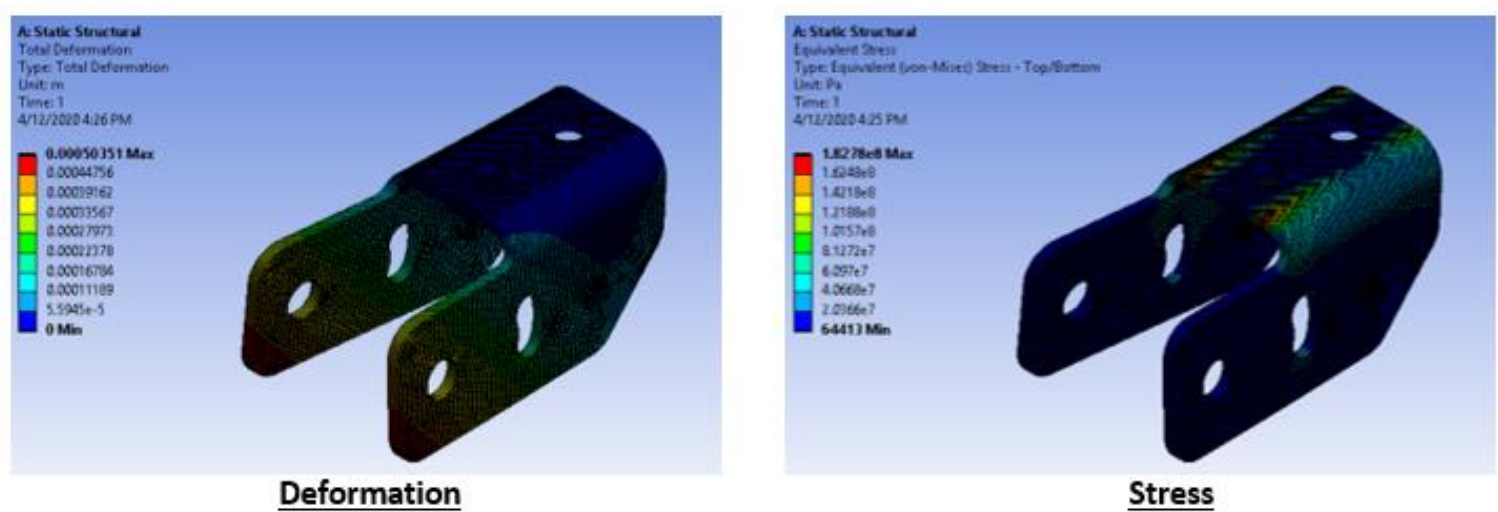

Note: We can see that the maximum stress $(182 \mathrm{Mpa})$ is less than the maximum allowable stress in our part (186 Mpa). This new stress value corresponds to a FOS $=1.5319$

Figure 8: Optimized body FEA 

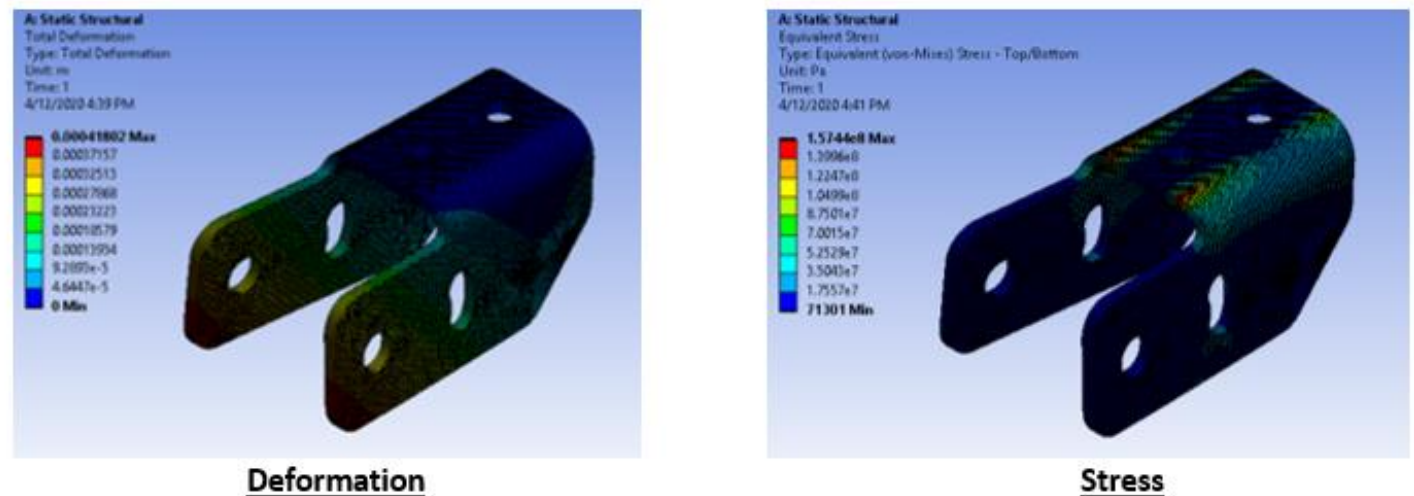

Note: We can see that the maximum stress ( $157 \mathrm{Mpa})$ is less than the maximum allowable stress in our part (186 Mpa). This new stress value corresponds to a FOS $=1.7784$

Figure 9: Optimized body FEA after modification

\section{Conclusion}

This research was carried out to optimize a sheet-metal part using direct optimization tool in ANSYS and analyze its effectiveness. Those factors are supposed to be design variables to which the output solution is highly sensitive thus they control the outcome. A direct link was established between the variation in design variables and mass of the part, which evidently showed us that they are linearly related. Following conclusions can be made as result of this study:

- Varying thickness of sheet is directly proportional to the mass of the body.

- Varying diameter of holes is inversely proportional to the mass of the body.

- Optimal part dimensions are not always ideal since they might be expensive to manufacture due to higher degree of precision.

The obtained results can serve as a theoretical background for designing and optimization process of various sheet-metal parts in industry, saving costs and time in manufacturing.

\section{References}

[1] Perks, H., Cooper, R. and Jones, C., 2005. Characterizing the role of design in new product development: An empirically derived taxonomy. Journal of product innovation management, 22(2), pp.111-127.

[2] Castorani, V., Vita, A., Mandolini, M. and Germani, M., 2017. A CAD-based method for multiobjectives optimization of mechanical products.Computer-Aided Design and Applications, 14(5), pp.563-571.

[3] Olhoff, N., Bendsøe, M.P. and Rasmussen, J., 1991. On CAD-integrated structural topology and design optimization. Computer Methods in Applied Mechanics and Engineering, 89(1-3), pp.259-279.

[4] Bendsøe, M.P. and Sigmund, O., 2004. Topology optimization by distribution of isotropic material. In Topology Optimization (pp. 1-69). Springer, Berlin, Heidelberg. 
[5] Fernández, E., Collet, M., Alarcón, P., Bauduin, S. and Duysinx, P., 2019. An aggregation strategy of maximum size constraints in density-based topology optimization. Structural and Multidisciplinary Optimization, 60(5), pp.2113-2130.

[6] Nocedal, J. and Wright, S., 2006. Numerical optimization. Springer Science \& Business Media.

[7] Buck, J.R. and Hancock, W.M., 1978. Manual optimization of ill-structured problems. International Journal of Man-Machine Studies, 10(2), pp.95-111.

[8] König, O. and Wintermantel, M., 2004. CAD-based evolutionary design optimization with CATIA V5. Proceedings of 1st Weimar Optimization and Stochastic Days WOST, Weimar, pp.130.

[9] Gunwant, D. and Misra, A., 2012. Topology Optimization of sheet metal brackets using ANSYS. MIT International Journal of Mechanical Engineering, 2(2), pp.120-126.

[10] Wang, M.Y., Wang, X. and Guo, D., 2003. A level set method for structural topology optimization. Computer methods in applied mechanics and engineering, 192(1-2), pp.227246.

[11] Fourie, P.C. and Groenwold, A.A., 2002. The particle swarm optimization algorithm in size and shape optimization. Structural and Multidisciplinary Optimization, 23(4), pp.259-267.

[12] Goldberg, D.E. and Deb, K., 1991. A comparative analysis of selection schemes used in genetic algorithms. In Foundations of genetic algorithms (Vol. 1, pp. 69-93). Elsevier.

[13] Bäck, T. and Schwefel, H.P., 1993. An overview of evolutionary algorithms for parameter optimization. Evolutionary computation, 1(1), pp.1-23.

[14] Grebenişan, G. and Salem, N., 2017. The multi-objective genetic algorithm optimization, of a superplastic forming process, using ansys ${ }^{\circledR}$. In MATEC Web of Conferences (Vol. 126, p. 03003). EDP Sciences.

[15] Fiandaca, G., Fraga, E.S. and Brandani, S., 2009. A multi-objective genetic algorithm for the design of pressure swing adsorption. Engineering Optimization, 41(9), pp.833-854.

[16] Huri, D. and Mankovits, T., 2019, October. Automotive rubber part design using machine learning. In IOP Conference Series: Materials Science and Engineering (Vol. 659, No. 1, p. 012022). IOP Publishing.

[17] Huri, D. and Mankovits, T., 2018, July. Comparison of the material models in rubber finite element analysis. In IOP Conference Series: Materials Science and Engineering (Vol. 393, No. 1, p. 012018). IOP Publishing.

[18] Mankovits, T., Szabó, T., Kocsis, I. and Páczelt, I., 2014. Optimization of the shape of axisymmetric rubber bumpers. Strojniški vestnik-Journal of Mechanical Engineering, 60(1), pp.61-71.

[19] Mankovits, T., Huri, D., Kállai, I., Kocsis, I. and Szabó, T., 2014. Material characterization and numerical simulation of a rubber bumper. International Journal of Mechanical, Aerospace, Industrial, Mechatronic and Manufacturing Engineering, 8(8), pp.1367-1370. 
[20] Mankovits, T., Kocsis, I., Portik, T., Szabó, T. and Páczelt, I., 2013. Shape design of rubber part using FEM. International Review of Applied Sciences and Engineering, 4(1), pp.85-94.

[21] Kocsis, I. and Mankovits, T., 2013. Application of non-parametric regression in engineering optimization. Analele Universitatii din Oradea Fasciola Management si Inginerie Tehnologica/Annals of the University of Oradea Fascicle of Management and Technological Engineering, 12(22), pp.159-162.

[22] Hafiz, A.A.M., Hussien, H.M.A., Gemeal, A.M.B. and Naranjel, V., TOPOLOGY OPTIMIZATION OF SHEET METAL PRODUCED USING COMBINATION DIE.

[23] Prasanna, P., Sushma, J. and Srikanth, K.P., 2018. Stress analysis and design optimization of a pressure vessel using Ansys package. Materials Today: Proceedings, 5(2), pp.4551-4562. 